\title{
Microvesicles from platelets: novel drivers of vascular inflammation
}

\author{
Tanja Vajen'; Sebastian F. Mause²; Rory R. Koenen ${ }^{1}$ \\ ${ }^{1}$ Department of Biochemistry, Cardiovascular Research Institute Maastricht (CARIM), Maastricht University, Maastricht, The Netherlands; ${ }^{2}$ Department of Cardiology, Medical Clinic \\ I, Medical Faculty, University Hospital RWTH Aachen, Aachen, Germany
}

\begin{abstract}
Summary
Microvesicles are receiving increased attention not only as biomarkers but also as mediators of cell communication and as integral effectors of disease. Platelets present a major source of microvesicles and release these microvesicles either spontaneously or upon activation. Platelet-derived microvesicles retain many features of their parent cells and have been shown to exert modulatory effects on vascular and immune cells. Accordingly, microvesicles from platelets can be measured at increased levels in patients with cardiovascular disease or individuals at risk. In addition, isolated microvesicles from platelets were
\end{abstract}

shown to exert immunomodulatory actions on various cell types. In this review the various aspects of platelet-derived microvesicles including release, clearance, measurement, occurrence during disease and relevance for the pathophysiology of vascular inflammation will be discussed.

\section{Keywords}

Platelet, microvesicle, microparticle, vascular inflammation, atherosclerosis

Received: November 19, 2014

Accepted after major revision: March 3, 2015

Epub ahead of print: May 21, 2015

http://dx.doi.org/10.1160/TH14-11-0962

Thromb Haemost 2015; 114: 228-236

Maastricht University

Maastricht, The Netherlands

Tel.: +3143 3881674, Fax: +31433884159

E-mail: r.koenen@maastrichtuniversity.nl

\section{Introduction}

Platelets release microvesicles upon activation and during prolonged storage. Although microvesicle release might play an important role in coagulation, evidence also points towards functions of these microvesicles in the functional modulation of immune cells. Microvesicles retain many properties of their parent cells, such as the repertoire of surface molecules, the ability to deposit chemokines to the vessel wall (1) and to confer inflammatory signals to distal target cells $(2,3)$. Here, we provide a brief state-ofthe-art review of the inflammatory function of platelets and platelet-derived microvesicles (PMVs) in particular.

\section{Pro-inflammatory roles of platelets}

Besides their role in haemostasis, platelets are receiving increased recognition as an integral component of the immune system (4). For example, platelets carry functional copies of toll-like receptor 4 (TLR4) enabling them to sense bacterial lipopolysaccharide (LPS) (5). Activation of platelet-TLR4 was found to induce binding of platelets to neutrophils, inducing the formation of neutrophil extracellular traps (NETs), which facilitated the entrapment of bacteria during sepsis (6). In addition, platelets rapidly respond to contact with bacteria and were recently found to cooperate with Kupffer cells to eradicate infection of B. cereus in the mouse liver (7). Yet besides the important functions in host defence, the action of platelets can also turn against the host, particularly during autoimmune disease. A detrimental role of platelets during the progression of atherosclerosis is well established (reviewed in $[8,9]$ ) and a recent study showed the presence of platelets in the spinal cord of mice with experimental autoimmune encephalomyelitis (EAE) and in lesions of patients with multiple sclerosis (10). Depletion of platelets was found to improve clinical scores of mice with induced EAE and reduced the inflammatory parameters in their spinal cords. Interestingly, platelets were found to play a role in the recruitment of leukocytes to the central nervous system after induction of EAE.

There are several possible mechanisms how platelets can facilitate the recruitment of leukocytes to sites of inflammation. First, activated platelets carry cytokines such as CD40L, which can directly induce endothelial activation through ligation of CD40 (11, 12 ), causing the production of adhesion molecules and chemokines.

Despite being anucleate, platelets retain the ability to translate mRNA leading, e.g. to the production of interleukin (IL) $1 \beta$ (13), a cytokine implicated in the inflammatory action of platelets to the vessel wall (14). Second, activated platelets can deposit chemokines onto the surface of inflamed endothelial cells, leading to recruitment of leukocytes. Most prominent examples are the chemokines CCL5 and CXCL4, which act in concert to attract monocytes, contributing to the progression of atherosclerosis $(15,16)$ and acute lung injury (17). Third, activated platelets avidly bind to leukocytes and modulate their activity. Platelet-monocyte interac- 
tion is initiated by P-selectin-PSGL-1 interactions and causes the transcription of inflammatory genes in monocytes (18). In addition, platelet binding considerably increases the adhesiveness of monocytes to endothelial cells by increasing the amount of possibilities of which an interaction with the endothelium can take place $(19,20)$. We have previously shown that CX3CR1, a chemokine receptor expressed by activated platelets mediated the complex formation of platelets and monocytes during hyperlipidaemia in mice and was involved in platelet-leukocyte interaction to vascular smooth muscle cells (SMC) (21). Besides acting on monocytes, also lymphocyte recruitment to the subendothelial matrix of a damaged vessel is specifically enhanced through the binding of platelets $(22,23)$. Genetic deletion of junctional adhesion molecule A in platelets causes the platelets to become hyperreactive (24), a condition that facilitates early atherosclerotic plaque formation in hyperlipidemic mice (25).

Finally, platelets contain many mitochondria that have been shown to be involved in platelet activation $(26,27)$. Extracellular mitochondria can be a source of damage-associated molecular patterns (DAMPs) mediating inflammation through their bacteria-like components (28). Recently it was demonstrated that thrombin-stimulated platelets release mitochondria into the extracellular milieu, either as free organelles or encapsulated within microvesicles (29). These mitochondria were shown to act as an endogenous substrates for phospholipase A2 IIA, which catalysed the release of inflammatory mediators that promoted leukocyte recruitment (29).

Thus, platelets contribute to inflammation in a variety of ways. Moreover, since platelets abundantly shed microvesicles either spontaneously or upon activation, they can transduce signals and modulate cellular functions not only in their local environment but also at a remarkable distance from their site of origin throughout the circulatory system.

\section{Release of microvesicles from platelets}

Although it is well established that many cells, particularly platelets, release microvesicles after activation (30), the mechanisms and the molecular factors that govern microvesicle formation are less clear. Early studies investigating platelets from patients with genetic defects have revealed a correlation between procoagulant activity and vesicle formation in platelets $(31,32)$. For example, platelets from individuals with Scott syndrome, a rare bleeding disorder, lack plasma membrane phosphatidylserine (PS) exposure upon activation due to a defect in membrane scrambling activity. The induction of a procoagulant PS-rich outer membrane leaflet is apparently also involved in the formation and release of vesicles, since Scott platelets did form little PMVs after stimulation with a variety of agonists (32). Platelets isolated from patients with Glanzmann's thrombasthenia, a genetic deficiency in glycoprotein (GP) IIbIIIa (integrin $\alpha I I b \beta 3$ ), or platelets treated with antibodies or compounds inhibiting integrin $\alpha I I b \beta 3$ showed a similar defect in PMVs generation (31). Other studies implied a role for the calpain class of cysteine proteases and the integrity of the platelet cy- toskeleton in the shedding of PMVs after platelet activation (33-35). For completion, it should be mentioned that platelets also release exosomes upon activation, which are small $(40-100 \mathrm{~nm})$ membrane-enclosed vesicles derived from organelles (36). These exosomes are outside the scope of this review. Interestingly, platelets do not require agonist-induced activation for the release of PMVs. The life span of platelets is regulated by an apoptosis-like process (37), and platelet concentrates destined for transfusion accumulate PMVs during prolonged storage (38). Similar to PMVs release by platelet activation, the integrity of the cytoskeleton and the action of integrin $\alpha \mathrm{IIb} \beta 3$ are important for this spontaneous release of PMVs, since inhibitors of integrin $\alpha \mathrm{IIb} \beta 3$ considerably attenuated PMVs release by stored platelets (38). Moreover, phenotypic differences exist between PMVs released upon activation and those released spontaneously during prolonged storage. Spontaneously released PMVs show a greater expression of CD42b compared to thrombin stimulated PMVs, whereas CD62P expression is increased on PMVs from thrombin-stimulated platelets. In addition, PMVs released spontaneously and by platelet sonication show a higher expression of activated $\alpha \operatorname{IIb} \beta 3$. Thrombin- and $\mathrm{Ca}^{2+}$ ionophore stimulated PMVs display a lower expression of $\alpha \mathrm{IIb} \beta 3$ but a higher expression of CD63 and annexin A5 (39). However, according to a later study there is no difference in binding to annexin A5, indicating that most PMVs express PS on their outer membrane (40). Moreover, different stimuli and activation pathways result not only in a variance of expression of surface molecules but also in the total number of released PMVs. Accordingly, the activation pathway determines the number and cargo of PMVs (41) and should be taken into account regarding functional studies on target cells.

Although platelets represent a major source of microvesicles in plasma, other cell types such as endothelial cells, smooth muscle cells, monocytes, lymphocytes and erythrocytes also release microvesicles. Despite differences in surface marker expression, the effects of microvesicles from these sources on vascular cells might overlap with those observed for PMVs and the effects might be net inflammatory, although microvesicles also mediate cell-cell communication (42). In addition, microvesicles from different cellular sources might act complementary in eliciting inflammatory reactions, e.g. as has been observed for microvesicles from atherosclerotic plaques (43). Extracellular vesicles from sources other than platelets, as well as exosomes and apoptotic bodies are outside of the scope of this review and have been discussed elsewhere $(3,44)$. Nevertheless, some notable aspects of these extracellular vesicles in vascular function are highlighted in $>$ Table 1.

\section{Clearance of microvesicles}

At present, a few studies investigated the clearance of microvesicles from the circulation. A rapid clearance of infused biotinylated PMVs was shown in a rabbit model (45). Recently, the clearance of PMVs from human apheresis platelet concentrates (PC) following platelet transfusion in severe thrombocytopenic patients was demonstrated. Following an immediate increase 


\begin{tabular}{lll}
\hline Cell origin & Effect & Ref. \\
\hline Endothelial cell & Endothelial and renal dysfunction & $(112,113)$ \\
& Vascular remodeling & $(114)$ \\
& Thrombosis, inflammation, endothelial activation & $(115,116)$ \\
& Endothelial cell survival & $(117)$ \\
& Angiogenesis & $(118,119)$ \\
& Smooth muscle cell phenotype & $(42)$ \\
& Dissociation of C-reactive protein & $(120)$ \\
\hline $\begin{array}{l}\text { Monocytes, lymphocytes, } \\
\text { plaque macrophages }\end{array}$ & Endothelial cell activation & $(43,121)$ \\
Smooth muscle cells & Increased thrombogenicity & $(122,123)$ \\
& Leukocyte adhesion to endothelial cells & $(109)$ \\
& Vascular calcification & $(124)$ \\
\hline
\end{tabular}

Table 1: Origin and effects of non-platelet extracellular microvesicles. after transfusion, PMVs declined with a half life of 5.8 and 5.3 hours for annexin A5 binding and CD61 exposing PMVs, respectively. Moreover, the clearance of PMVs was faster compared to platelets (46). The underlying mechanism of the clearance from the circulation is not thoroughly investigated. However, recently it has been shown that lactadherin (also termed MFG-E8) is an important mediator for clearance of PMVs. Lactadherindeficient mice showed an increased number of PMVs in plasma and a decreased phagocytosis by macrophages compared to wild type mice (47). Moreover, developmental endothelial locus-1 (Del-1), a glycoprotein homologue of lactadherin secreted by endothelial cells has also been shown to be involved in the clearance of PS-expressing microvesicles (48). Regarding the roles of PMVs in vascular inflammation and coagulation, the clearance of PMVs from the circulation might be an important process, which merit further investigation.

\section{Isolation and storage of platelet microvesicles for analysis}

It is still challenging to achieve an accurate determination of PMVs since their characteristics and levels were found to depend on a large number of variables such as the manner of blood collection, anticoagulation, plasma preparation, sample isolation, and storage as well as the detection method (49-51). Blood is commonly collected with large bore needle to minimise shear forces and sodium citrate supplemented with dextrose (ACD) are common anticoagulants for PMVs analysis (52). Several differential centrifugation procedures have been established. Most commonly, platelet-poor plasma (PPP) is prepared by low-speed centrifugation and PMVs are subsequently pelleted by a high-speed centrifugation step. However, by following these two steps, some residual platelets might be present, which can be removed by filtration (see below) or a second high speed centrifugation step, prior to the pelleting of the PMVs (49). Yet several studies have reported a $50-80 \%$ decrease in microvesicle count due to this second centrifugation $(53,54)$ as PMVs might be lost during disposal of the supernatant fractions. Thus PMVs might rather be detected directly in PPP to prevent a potential loss of PMVs during sample processing.
The International Society of Extracellular Vesicles (ISEV) recommends to isolate microvesicles after depletion of platelets, cells and large apoptotic bodies by one or more centrifugation and/or size exclusion steps by pelleting microvesicles by centrifugal force of 10,000-20,000g (55). Impurities of residual platelets, PMVs aggregates and other contaminants can then be removed from the resuspended pellet by filtration ( $0.8 \mu \mathrm{m}$ pore size). During every centrifugation step, all variables, i.e. g-force, rotor type and time should be properly selected to minimise contamination risk as well as loss of sample material. The microvesicle isolation from plasma should proceed immediately after blood collection. It must be noted that fresh plasma, particularly from patients with autoimmune inflammatory disease, contains soluble antigens that might contribute to the formation of immune complexes, which might co-purify with microvesicles (56). In practice, however, clinical studies often require the storage of plasma samples. Freezing of samples that contain blood cells and/or platelets results in the formation of microvesicle-like cellular fragments during thawing $(50,53,57)$. Therefore it might be considered to remove blood cells and platelets prior to storage, preferably by snap freezing in liquid nitrogen and storage at $-80^{\circ} \mathrm{C}$. Freezing and thawing may affect the number and characteristics of PMVs, although this is somewhat controversial as previous studies reported that freeze-thawing of double-centrifuged plasma results in increased levels of PMVs (54), whereas others have reported that microvesicles counts do not significantly differ in double-centrifuged plasma before and after thawing $(58,59)$. A prolonged storage results in a decreased number of PMVs and the loss of specific surface molecules (50), possibly through the action of protease activity that accompanied platelet activation (60). Taken together, freeze-thaw cycles should be minimised and the use of freshly isolated PMVs from plasma is recommended for analysis of PMVs.

\section{Quantitation and characterisation of platelet microvesicles}

Various quantitative and qualitative methods have been established for the analysis of PMVs. Flow cytometry is most commonly used for the qualitative and quantitative characterisation of microvesicles. Concerning their small heterogeneous size, the 
quantification of PMVs remains challenging. Still useful methods have been established. The flow cytometer used for PMVs analysis should have a low size detection threshold. Newer instruments i.e. Gallios (Beckman-Coulter, Pasadena, CA, USA), BD-Influx (BD Biosciences, Franklin Lakes, NJ, USA) and Apogee (Apogee Flow Systems Ltd., Hemel Hempstead, UK) enable the discrimination of particles of $100-200 \mathrm{~nm}$ in size $(61,62)$. The conventional method uses antibodies against annexin A5 and specific cell surface molecules, i.e. integrin aIIb (GPIIb, CD41a). A recent study demonstrates that most PMVs express PS on their outer membrane, suggesting that annexin A5 is sufficient as a marker for quantification (40). Further, microbeads (e.g. Megamix ${ }^{\mathrm{TM}}$, Biocytex, Marseille, France or SPHERO ${ }^{\mathrm{TM}}$ AccuCount, Spherotech, Lake Forest, IL, USA) with a defined size and count are used to calculate the absolute cell number of PMVs. The International Society on Thrombosis and Haemostasis (ISTH) has developed a standardised method for the quantification of microvesicles that uses the Megamix ${ }^{\mathrm{TM}}$ solution (63). Another possibility is to couple PMVs to micrometre-sized latex beads using antibodies against specific surface markers, similar to that done for exosomes (64).

The results of flow cytometry analysis depend on analytical factors i.e. the type of instrument, instrument settings and the resolution but also on factors related to the sample preparation and storage. Other state-of-the-art technologies include dynamic light scattering (DLS), which calculates the differential size distribution of a microvesicle population. Nanoparticle tracking analysis (NTA) and resistive pulse sensing (RPS) determine the absolute size distribution $(65,66)$. Additionally, Raman spectroscopy can provide information about the biochemical composition of microvesicles (67). Further methods have been applied and adapted for the assessment of microvesicles. Electron microscopy (EM) and transmission electron microscopy (TEM) allows analysing the morphology, size and presence of specific surface molecules (68). Advances in electron microscopy might enable visualisation of their three-dimensional structures at high magnification (69). Moreover, atomic force microscopy (AFM) has been applied for the assessment of microvesicles morphology in a nanometer resolution $(56,70)$. Other techniques include small-angle X-ray scattering or size-exclusion chromatography combined with dynamic light scattering, both of which are useful for size determination (68).

Among the quantification of isolated microvesicles, there is also the possibility to measure microvesicles directly in plasma. Capture-based assays, in which microtiter wells are coated with a probe, e. g. annexin A5, for the binding of negatively charged phospholipids or antibodies against specific surface markers can be used to quantify microvesicles via their procoagulant activity or through peroxidase conjugated-antibodies, respectively (71). The main advantage of these assays is a fast and direct measurement of PMVs in plasma. However, these methods do not reveal any information about the size and absolute numbers of PMVs. In addition, problems with the interpretation of the results might occur because not all PMVs might express negatively charged phospholipids or platelet surface markers. Thus, recent studies suggested the use of lactadherin rather than annexin $\mathrm{V}$, as it represents a more sensitive and calcium-independent probe for detection of PS (72). Thus, a major future goal is to standardise the isolation and quantification method of microvesicles to ascertain reproducibility and comparability.

\section{Platelet microvesicles in patients with cardiovascular disease}

Microvesicles are arousing interest not only as potential biomarkers for inflammatory diseases but also due to their role in inflammatory diseases. For example, PMVs have been implicated in the development and progression of atherosclerosis, the underlying cause for cardiovascular diseases (CVD)(73). Increased circulating levels of PMVs were found in patients with clinically severe peripheral atherosclerosis $(74,75)$. Moreover, increased PMVs levels were not only detected in diseased patients, but also in older healthy subjects (76). The main risk factors for atherosclerosis, including diabetes mellitus type I and II, hypertension, hyperlipidaemia, hypercholesterolaemia, sleep apnoea and obesity/metabolic syndrome have all been associated with increased PMVs levels (77-83). Increased levels of PMVs identified in patients with metabolic syndrome positively correlated with waist circumference, glycaemia and oxidative stress markers, e.g. glutathione peroxidase and urinary isoprostane (77). Interestingly, the microvesicle phenotype might vary within the type of diabetes. In patients with diabetes type I, increased levels of annexin A5- and CD41-positive microvesicles have been reported, whereas in type II diabetes only annexin A5-positive microvesicles were significantly increased (79). A recent study shows the relation of the type of plaque, with circulating microparticle number, phenotype and activation status of the originating cell in familial hypercholesterolaemia (FH) patients (84). There, increased levels of circulating microvesicles, in particular lymphocyte and monocyte-derived microvesicles were identified in patients with $\mathrm{FH}$ with subclinical lipid-rich atherosclerotic plaques (84). In particular, circulating $\mathrm{CD} 5^{+} / \mathrm{CD}^{+}$-lymphocyte microvesicles were found in patients with a lipid-rich atherosclerotic plaque but not in patients with a calcified atherosclerotic plaque. The standard treatment in hypercholesterolaemia and hyperlipidaemia is the lipid-lowering treatment (LLT) with statins. The influence of statins on the number and properties of microvesicles remains controversial. However, hypercholesterolaemic patients who received statins have a reduced level of circulating microvesicles and cell activation markers originating from platelets, endothelial cells and leukocytes (83). Moreover, the administration of atorvastatin reduced thrombin generation and expression of tissue factor, GPIIIa and P-selectin on PMVs in patients with peripheral vascular disease (85). Finally, it remains unclear whether the effects are due to a microvesicledependent mechanism or due to the reduction of the lipoprotein levels or inflammatory markers. Women after menopause have an increased risk for CVD. One study reported increased levels of PMVs associated with an early stage coronary artery calcification in menopausal women (86). As a whole, elevated levels of PMVs may reflect "vulnerable blood", referring to a hypercoagulable state 
with increased blood thrombogenicity and augmented risk for atherothrombotic events (75). According to such a concept, evaluation of PMVs may help to identify patients at risk for such events and for an adverse outcome. However, the heterogeneity of microvesicles might hamper a clear connection between numbers of circulating microvesicles from a particular cellular origin and the disease risk or clinical severity (87).

\section{Interaction and modulation of vascular and immune cells by platelet microvesicles}

Since PMVs carry a surface molecule repertoire similar (but not identical) to that of their parent cells, there are ample opportunities for interaction with other cells ( $>$ Figure 1). For example,
PMVs might interact with endothelial cells via interactions of GPIb (in complex with GPIX and GPV) or integrin $\alpha \mathrm{IIb} \beta 3$ with VWF presented on the endothelial surface or subendothelial matrix. Accordingly, PMVs were found to adhere both to the subendothelium and to activated endothelial cells and recruited activated platelets to the injured endothelium (88). In a later study, isolated PMVs exogenously added to blood were shown to increase the thrombogenicity of platelets under flow both on a collagen surface as well as on perfused aortic specimen from pigs or individuals with atherosclerotic plaques (89). By the release of PMVs, platelets may engage a functional autocrine, self-amplifying loop mechanism to substantiate and disseminate their procoagulatory potential (87). Isolated microvesicles from individuals with metabolic syndrome consisted largely of PMVs and caused dysfunction of cultured endothelial cells as well as hypocontractility

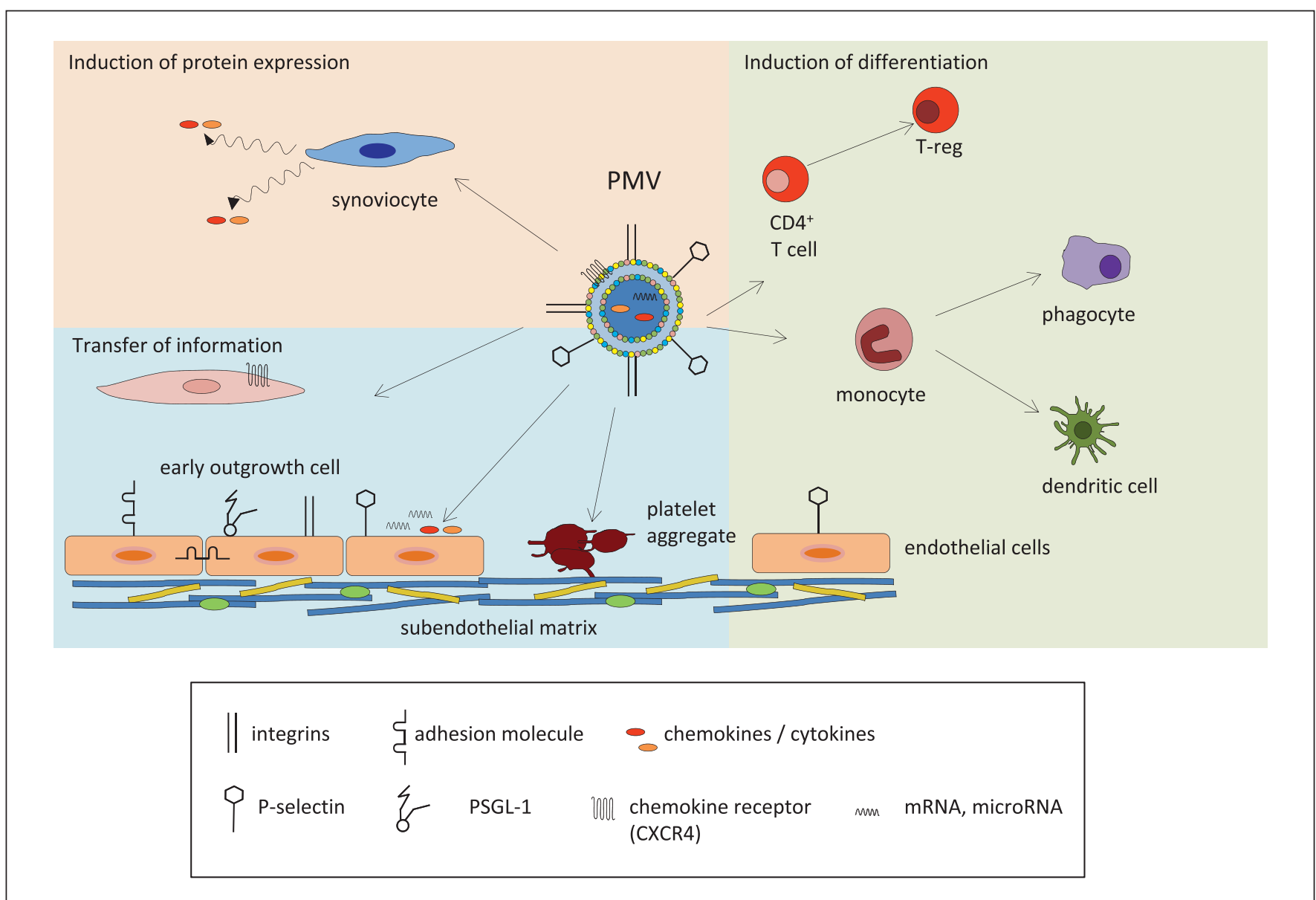

Figure 1: Influence of platelet microvesicles: induction of protein expression, induction of cell differentiation and transfer of information on target cells. PMVs interact with the injured endothelium or subendothelial matrix via integrins, thereby being able to recruit activated platelets and increasing thrombogenicity $(87,89)$. Further, PMVs transfer and deposit inflammatory mediators such as CCL5 onto the endothelial surface (1). Upon contact with T lymphocytes, PMVs induce differentiation of naïve CD4 ${ }^{+}$T-cells into Foxp3 ${ }^{+}$regulatory T cells (93). In addition, PMVs induce the expression of proinflammatory cytokines and chemokines, i. e. IL-6, CXCL1 and CXCL8 in synoviocytes, a cell type important in the pathophysiology of rheumatoid arthritis (96). Likewise, PMVs transfer the chemokine receptor CXCR4 to angiogenic early outgrowth cells, inducing the release of proangiogenic factors and increase their vasoregenerative potential (103). Co-incubation of PMVs and monocytes induce a phenotypic shift of surface markers towards a phagocytic phenotype (108). However, PMVs might also induce the differentiation of monocytes into immature dendritic cells, resulting in a reduced inflammatory and phagocytic potential (110). Finally, PMVs can be involved in internalisation and the transfer of nucleic acids including mRNA and microRNA $(104,106)$. 
Table 2: Interaction and effects of plateletderived microvesicles on target cells.

\begin{tabular}{|c|c|c|}
\hline Target cell type & Effect & Ref. \\
\hline $\begin{array}{l}\text { Early outgrowth cells } \\
\text { (EOCs) }\end{array}$ & $\begin{array}{l}\text { Endothelial regeneration by recruitment, migration, differentiation } \\
\text { of EOC }\end{array}$ & (103) \\
\hline Endothelial cell & $\begin{array}{l}\text { Deposition and transfer of chemokines } \\
\text { Transfer of nucleic acids }\end{array}$ & $\begin{array}{l}(1),(87) \\
(104,106)\end{array}$ \\
\hline Monocyte & Induction of differentiation to dendritic cell & $(110)$ \\
\hline Monocyte & Modulation of monocyte/endothelial interaction & $(107)$ \\
\hline $\begin{array}{l}\text { Monocyte/- } \\
\text { Macrophage }\end{array}$ & $\begin{array}{l}\text { Modulation of monocyte phenotype / Induction of differentiation } \\
\text { into phagocyte }\end{array}$ & $\begin{array}{l}(108,111) \\
(40)\end{array}$ \\
\hline Platelets & Modulation of thrombogenesis & (40) \\
\hline Platelets & Increase of thrombogenicity & (89) \\
\hline Platelets & Platelet deposition in atherosclerotic plaque & (89) \\
\hline Subendothelium & $\begin{array}{l}\text { Platelet interaction with subendothelial matrix, platelet deposition } \\
\text { and thrombus formation }\end{array}$ & $(88,89)$ \\
\hline Synoviocyte & Inflammatory response in rheumatoid arthritis & (96) \\
\hline T cell & Differentiation to Foxp3 ${ }^{+} T_{\text {reg }}$ cells & (93) \\
\hline
\end{tabular}

and the production of reactive oxygen species in mouse aortic explants $(90,91)$. During rolling interactions with endothelial cells, PMVs can deposit inflammatory mediators such as CCL5 onto the endothelial surface, leading to subsequent recruitment of mononuclear cells (1). These rolling interactions appeared to depend largely on P-selectin and GPIb, while the actual transfer of CCL5 rather depended on integrin $\alpha \operatorname{IIb} \beta 3$ and junctional adhesion molecule A. This process might play a role particularly in early phases of atherosclerotic lesion formation, during which endothelial dysfunction is propagated by activated platelets (8). T lymphocytes are among the earliest cells that are recruited in the atherosclerotic lesion (92). Recently, an immunomodulatory role of PMVs on $\mathrm{CD}^{+} \mathrm{T}$ cells has been described. Spontaneously released PMVs from platelets (termed „ectosomes" in the study) induced the differentiation of naïve $\mathrm{CD} 4^{+} \mathrm{T}$ cells into Foxp $3^{+}$regulatory T cells. These effects were dependent on platelet-derived TGF- $\beta$. In addition, PMVs decreased the release of IFN $\gamma$, TNF $\alpha$ and IL- 6 after incubation with $\mathrm{T}$ cells. Thus, the interaction of PMVs with $\mathrm{CD} 4^{+} \mathrm{T}$ cells points towards an immunosuppressive response in vitro (93).

During later phases, PMVs might also play a role by inducing the proliferation of vascular SMC (94). Since SMC are able to express the chemokine CX3CL1 (95), the functional interaction of SMC with PMVs might be mediated through CX3CR1 present on PMVs $(1,21)$. Similar to platelets, the CX3CL1-CX3CR1 axis might also be involved in the binding of PMVs to monocytes (21). Yet PMVs also appear to play an important role in other autoimmune diseases, such as rheumatoid arthritis (RA). In an elegant study, Boilard and colleagues demonstrated that platelet depletion delayed the onset and ameliorated the symptoms of RA in mice (96). Interestingly, large numbers of microvesicles, the majority carrying the platelet marker CD41a, were measured in synovial fluid from patients with RA but not osteoarthritis. Platelets abundantly released PMVs when brought into contact with cultured fibroblast-like synoviocytes, a cell type important in the pathophysi- ology of RA. Notably, contact with PMVs induced the production of cytokines, e. g. IL- 6 and the neutrophil-attracting chemokines CXCL1 and CXCL8 in fibroblast-like synoviocytes. However PMV preparations, particular from patients with auto-immune disease, might also contain immune complexes with similar physicochemical properties as PMVs (56). Those immune complexes might thus contaminate the PMV preparations and have been shown to induce trigger inflammatory responses $(97,98)$. On the other hand, it has been demonstrated that microvesicles actively present immunoglobulins on their surface in specimen of patients with RA $(99,100)$. In addition, a recent study identified platelet microparticle-associated immune complexes in RA synovial fluid, which might be formed by recognition of citrullinated autoantigens such as vimentin and fibrinogen (101). These platelet microparticle-associated immune complexes have been identified as bioactive pro-inflammatory molecules eliciting leukotriene release by neutrophils. Taken together, the above studies highlight the inflammatory action of platelets in inflammatory disease and emphasise the role of PMVs as active conveyors of inflammatory information. This transfer of information can occur on several levels, e.g. functional surface receptors might be carried from PMVs to target cells, as demonstrated for CXCR4, the receptor for the chemokine CXCL12. Platelets activated with collagen and thrombin release PMVs that are rich in CXCR4 (102). Incubation of cells that did not express CXCR4 with PMVs acquired the functional receptor and became prone to infection with T-tropic human immunodeficiency strains. Likewise, PMVs transferred functional CXCR4 to angiogenic early outgrowth cells, induce the release of pro-angiogenic factors and increase their vasoregenerative potential (103). The mechanism of cellular modulation by PMVs is not completely known at present. Besides a role of active molecules in PMVs, it might also involve internalisation and a possible transfer of nucleic acids including mRNA and microRNA from the PMVs to the target cell (104-106). 
Already in the 1990s, PMVs were shown to have a stimulating effect on monocytic cells, inducing the expression of inflammatory surface markers and enhancing chemotaxis (107). We have shown that incubation of monocytic cells and primary monocytes with PMVs isolated from stored quiescent platelets induced a phenotypic shift of surface markers towards a phagocytic phenotype (108). In addition, the expression of matrix metalloprotease 9, hydrogen peroxide and pro-inflammatory factors, including C $5 \mathrm{a}$ and tumour necrosis factor (TNF) a was strongly increased after incubation with PMVs. Moreover, PMVs isolated from atherosclerotic lesions have been shown to stimulate monocyte adhesion to the endothelium (109) and PMVs increased the expression of ICAM-1 in monocytes (40). The above findings appear to conflict with a simultaneous study, where spontaneously released PMVs rather attenuated the release of TNFa from zymosan-activated macrophages and modified the differentiation of monocytes into dendritic cells, resulting in cells with reduced inflammatory and phagocytic potential (110). Accordingly, the manner of platelet activation/PMVs formation was found to determine the effects of the resulting PMVs on monocytic cells $(40,111)$. This again emphasizes that the heterogeneity of microvesicles, even when derived from a single cell type considerably influences the downstream effects on target cells, and should be taken into account when designing functional experiments. An overview of the interactions and effects of PMVs on target cells is summarised in $\mathbf{T}$ Table 2.

\section{Conclusion}

During the past decade, the field of extracellular vesicles has been receiving increasing attention. Since platelets are a major source of microvesicles, PMVs are expected to play a prominent role in this emerging new area. However, various aspects still need attention. First, the measurement of PMVs in biologic specimen is still hampered by their small size, emphasizing the necessity of equipment capable of detecting small particulate species. Second, the heterogeneity of PMVs, possibly due to the variety of stimuli that lead to PMVs generation might complicate their utilisation as biomarkers as well as the characterisation of their influence on other cells. Third, many of the pathophysiological effects attributed to PMVs are derived from laboratory experiments using cultured cells. Disease models investigating the role of PMVs-formation in vivo are still difficult to establish and limited to transfusion of isolated PMVs. Moreover, genetic defects that affect PMVs formation often lead to more general defects in platelet function (e.g. integrin $\alpha \operatorname{IIb} \beta 3$-deficiency). Nevertheless, the current evidence of the importance of PMVs in autoimmune and cardiovascular disease is convincing and the interesting field of platelet extracellular vesicles will continue to expand.

\section{Acknowledgments}

This work was supported by Deutsche Forschungsgemeinschaft (DFG Ko2948/1-2, SFB1123/1 A02 and FOR809) and the Netherlands Foundation for Scientific Research (ZonMW VIDI 016.126.358) awarded to R.R.K.

\section{Conflicts of interest}

None declared.

\section{References}

1. Mause SF, et al. Platelet microparticles: a transcellular delivery system for RANTES promoting monocyte recruitment on endothelium. Arterioscler Thromb Vasc Biol 2005; 25: 1512-1518.

2. Mause SF, Weber C. Microparticles: protagonists of a novel communication network for intercellular information exchange. Circ Res 2010; 107: 1047-1057.

3. Thery C, et al. Membrane vesicles as conveyors of immune responses. Nat Rev Immunol 2009; 9: 581-593.

4. Rondina MT, et al. Platelets as cellular effectors of inflammation in vascular diseases. Circ Res 2013; 112: 1506-1519.

5. Andonegui G, et al. Platelets express functional Toll-like receptor-4. Blood 2005; 106: 2417-2423.

6. Clark SR, et al. Platelet TLR4 activates neutrophil extracellular traps to ensnare bacteria in septic blood. Nat Med 2007; 13: 463-469.

7. Wong $\mathrm{CH}$, et al. Nucleation of platelets with blood-borne pathogens on Kupffer cells precedes other innate immunity and contributes to bacterial clearance. Nat Immunol 2013; 14: 785-792.

8. Gawaz M, et al. Platelets in inflammation and atherogenesis. J Clin Invest 2005; 115: 3378-3384.

9. Weber C. Platelets and chemokines in atherosclerosis: partners in crime. Circ Res 2005; 96: 612-616.

10. Langer HF, et al. Platelets contribute to the pathogenesis of experimental autoimmune encephalomyelitis. Circ Res 2012; 110: 1202-1210.

11. Henn V, et al. CD40 ligand on activated platelets triggers an inflammatory reaction of endothelial cells. Nature 1998; 391: 591-594.

12. Lievens D, et al. Platelet CD40L mediates thrombotic and inflammatory processes in atherosclerosis. Blood 2010; 116: 4317-4327.

13. Lindemann $S$, et al. Activated platelets mediate inflammatory signaling by regulated interleukin 1beta synthesis. J Cell Biol 2001; 154: 485-490.

14. Gawaz M, et al. Platelets induce alterations of chemotactic and adhesive properties of endothelial cells mediated through an interleukin-1-dependent mechanism. Implications for atherogenesis. Atherosclerosis 2000; 148: 75-85.

15. Huo $\mathrm{Y}$, et al. Circulating activated platelets exacerbate atherosclerosis in mice deficient in apolipoprotein E. Nat Med 2003; 9: 61-67.

16. Koenen RR, et al. Disrupting functional interactions between platelet chemokines inhibits atherosclerosis in hyperlipidemic mice. Nat Med 2009; 15: 97-103.

17. Grommes J, et al. Disruption of platelet-derived chemokine heteromers prevents neutrophil extravasation in acute lung injury. Am J Respir Crit Care Med 2012; 185: 628-636.

18. Weyrich AS, et al. Monocyte tethering by P-selectin regulates monocyte chemotactic protein-1 and tumor necrosis factor-alpha secretion. Signal integration and NF-kappa B translocation. J Clin Invest 1995; 95: 2297-2303.

19. Theilmeier G, et al. Circulating activated platelets assist THP-1 monocytoid/endothelial cell interaction under shear stress. Blood 1999; 94: 2725-2734.

20. da Costa Martins P, et al. Platelet-monocyte complexes support monocyte adhesion to endothelium by enhancing secondary tethering and cluster formation. Arterioscler Thromb Vasc Biol 2004; 24: 193-199.

21. Postea O, et al. Contribution of platelet CX(3)CR1 to platelet-monocyte complex formation and vascular recruitment during hyperlipidemia. Arterioscler Thromb Vasc Biol 2012; 32: 1186-1193.

22. $\mathrm{Hu} \mathrm{H}$, et al. Platelets enhance lymphocyte adhesion and infiltration into arterial thrombus. Thromb Haemost 2010; 104: 1184-1192.

23. Spectre G, et al. Platelets selectively enhance lymphocyte adhesion on subendothelial matrix under arterial flow conditions. Thromb Haemost 2012; 108: 328-337.

24. Naik MU, et al. JAM-A protects from thrombosis by suppressing integrin alphaIIbbeta3-dependent outside-in signaling in platelets. Blood 2012; 119: 3352-3360.

25. Karshovska E, et al. Hyperreactivity of junctional adhesion molecule a-deficient platelets accelerates atherosclerosis in hyperlipidemic mice. Circ Res 2015; 116 : 587-599. 
26. Liu F, et al. Mitochondrially mediated integrin alphaIIbbeta3 protein inactivation limits thrombus growth. J Biol Chem 2013; 288: 30672-30681.

27. Jobe SM, et al. Critical role for the mitochondrial permeability transition pore and cyclophilin D in platelet activation and thrombosis. Blood 2008; 111: $1257-1265$.

28. Zhang Q, et al. Circulating mitochondrial DAMPs cause inflammatory responses to injury. Nature 2010; 464: 104-107.

29. Boudreau LH, et al. Platelets release mitochondria serving as substrate for bactericidal group IIA-secreted phospholipase A2 to promote inflammation. Blood 2014; 124: 2173-2183.

30. Morel O, et al. Mechanisms of microparticle generation: on the trail of the mitochondrion! Semin Thromb Hemost 2010; 36: 833-844.

31. Gemmell $\mathrm{CH}$, et al. Platelet-derived microparticle formation involves glycoprotein IIb-IIIa. Inhibition by RGDS and a Glanzmann's thrombasthenia defect. J Biol Chem 1993; 268: 14586-14589.

32. Sims PJ, et al. Assembly of the platelet prothrombinase complex is linked to vesiculation of the platelet plasma membrane. Studies in Scott syndrome: an isolated defect in platelet procoagulant activity. J Biol Chem 1989; 264: 17049-17057.

33. Yano $Y$, et al. The effects of calpeptin (a calpain specific inhibitor) on agonist induced microparticle formation from the platelet plasma membrane. Thromb Res 1993; 71: 385-396.

34. Pasquet JM, et al. Calcium influx is a determining factor of calpain activation and microparticle formation in platelets. Eur J Biochem 1996; 239: 647-654.

35. Yano Y, et al. The role of protein phosphorylation and cytoskeletal reorganisation in microparticle formation from the platelet plasma membrane. Biochem J 1994; 299: 303-308.

36. Heijnen HF, et al. Activated platelets release two types of membrane vesicles: microvesicles by surface shedding and exosomes derived from exocytosis of multivesicular bodies and alpha-granules. Blood 1999; 94: 3791-3799.

37. Kile BT. The role of apoptosis in megakaryocytes and platelets. Br J Haematol 2014; 165: 217-226.

38. Cauwenberghs $\mathrm{S}$, et al. Shedding of procoagulant microparticles from unstimulated platelets by integrin-mediated destabilisation of actin cytoskeleton. FEBS Lett 2006; 580: 5313-5320.

39. Vasina EM, et al. Aging- and activation-induced platelet microparticles suppress apoptosis in monocytic cells and differentially signal to proinflammatory mediator release. Am J Blood Res 2013; 3: 107-123.

40. Montoro-Garcia S, et al. Small-size platelet microparticles trigger platelet and monocyte functionality and modulate thrombogenesis via P-selectin. $\mathrm{Br} \mathrm{J} \mathrm{Hae}-$ matol 2014; 166: 571-580.

41. Aatonen MT, Ohman T, Nyman TA, et al. Isolation and characterisation of platelet-derived extracellular vesicles. J Extracell Vesicles 2014; Epub ahead of print.

42. Hergenreider E, et al. Atheroprotective communication between endothelial cells and smooth muscle cells through miRNAs. Nat Cell Biol 2012; 14: 249-256.

43. Leroyer AS, et al. CD40 ligand+ microparticles from human atherosclerotic plaques stimulate endothelial proliferation and angiogenesis a potential mechanism for intraplaque neovascularisation. J Am Coll Cardiol 2008; 52: 1302-1311.

44. Loyer X, et al. Microvesicles as cell-cell messengers in cardiovascular diseases. Circ Res 2014; 114: 345-353.

45. Rand ML, et al. Rapid clearance of procoagulant platelet-derived microparticles from the circulation of rabbits. J Thromb Haemost 2006; 4: 1621-1623.

46. Rank A, et al. Clearance of platelet microparticles in vivo. Platelets 2011; 22: 111-116.

47. Dasgupta SK, Abdel-Monem H, Niravath P, et al. Lactadherin and clearance of platelet-derived microvesicles. Blood 2009; 113: 1332-1339.

48. Dasgupta SK, et al. Developmental endothelial locus-1 (Del-1) mediates clearance of platelet microparticles by the endothelium. Circulation 2012; 125 : 1664-1672.

49. Yuana Y, et al. Pre-analytical and analytical issues in the analysis of blood microparticles. Thromb Haemost 2011; 105: 396-408.

50. Dey-Hazra E, et al. Detection of circulating microparticles by flow cytometry: influence of centrifugation, filtration of buffer, and freezing. Vasc Health Risk Manag 2010; 6: 1125-1133.

51. Shantsila E, et al. Circulating microparticles: challenges and perspectives of flow cytometric assessment. Thromb Haemost 2014; 111: 1009-1014.
52. Jy W, et al. Measuring circulating cell-derived microparticles. J Thromb Haemost 2004; 2: 1842-1851.

53. Ayers L, et al. Measurement of circulating cell-derived microparticles by flow cytometry: sources of variability within the assay. Thromb Res 2011; 127: 370-377.

54. van Ierssel SH, et al. Flow cytometric detection of endothelial microparticles (EMP): effects of centrifugation and storage alter with the phenotype studied. Thromb Res 2010; 125: 332-339.

55. Witwer KW, et al. Standardisation of sample collection, isolation and analysis methods in extracellular vesicle research. J Extracell Vesicles 2013; Epub ahead of print.

56. Gyorgy B, et al. Detection and isolation of cell-derived microparticles are compromised by protein complexes resulting from shared biophysical parameters. Blood 2011; 117: e39-48.

57. Mobarrez F, et al. A multicolor flow cytometric assay for measurement of platelet-derived microparticles. Thromb Res 2010; 125: e110-116.

58. Berckmans RJ, et al. Cell-derived microparticles circulate in healthy humans and support low grade thrombin generation. Thromb Haemost 2001; 85: 639-646.

59. Chandler WL. Microparticle counts in platelet-rich and platelet-free plasma, effect of centrifugation and sample-processing protocols. Blood Coagul Fibrinolysis 2013; 24: 125-132.

60. Qiao JL, et al. Proteolysis of platelet receptors in humans and other species. Biol Chem 2010; 391: 893-900.

61. Lacroix R, et al. Overcoming limitations of microparticle measurement by flow cytometry. Semin Thromb Hemost 2010; 36: 807-818.

62. van der Vlist EJ, et al. Fluorescent labeling of nano-sized vesicles released by cells and subsequent quantitative and qualitative analysis by high-resolution flow cytometry. Nat Protoc 2012; 7: 1311-1326.

63. Lacroix R, et al. Standardisation of platelet-derived microparticle enumeration by flow cytometry with calibrated beads: results of the International Society on Thrombosis and Haemostasis SSC Collaborative workshop. J Thromb Haemost 2010; 8: 2571-2574.

64. Thery C, et al. Isolation and characterisation of exosomes from cell culture supernatants and biological fluids. Curr Protoc Cell Biol 2006; Chapter 3: Unit 3 22.

65. Vogel R, et al. Quantitative sizing of nano/microparticles with a tunable elastomeric pore sensor. Anal Chem 2011; 83: 3499-3506.

66. Dragovic RA, et al. Sizing and phenotyping of cellular vesicles using Nanoparticle Tracking Analysis. Nanomed 2011; 7: 780-788.

67. Bell NC, et al. Emerging techniques for submicrometer particle sizing applied to Stober silica. Langmuir 2012; 28: 10860-10872.

68. Varga Z, et al. Towards traceable size determination of extracellular vesicles. J Extracell Vesicles 2014; Epub ahead of print.

69. Harapin J, et al. Structural analysis of supramolecular assemblies by cryo-electron tomography. Structure 2013; 21: 1522-1530.

70. Yuana Y, et al. Atomic force microscopy: a novel approach to the detection of nanosized blood microparticles. J Thromb Haemost 2010; 8: 315-323.

71. Ueba T, et al. Level, distribution and correlates of platelet-derived microparticles in healthy individuals with special reference to the metabolic syndrome. Thromb Haemost 2008; 100: 280-285.

72. Nielsen MH, et al. A flow cytometric method for characterisation of circulating cell-derived microparticles in plasma. J Extracell Vesicles 2014; Epub ahead of print.

73. Amabile N, et al. Microparticles: key protagonists in cardiovascular disorders. Semin Thromb Hemost 2010; 36: 907-916.

74. Tan KT, et al. Platelet microparticles and soluble P selectin in peripheral artery disease: relationship to extent of disease and platelet activation markers. Ann Med 2005; 37: 61-66.

75. Namba M, et al. Circulating platelet-derived microparticles are associated with atherothrombotic events: a marker for vulnerable blood. Arterioscler Thromb Vasc Biol 2007; 27: 255-256.

76. van der Zee PM, et al. P-selectin- and CD63-exposing platelet microparticles reflect platelet activation in peripheral arterial disease and myocardial infarction. Clin Chem 2006; 52: 657-664.

77. Cohen Z, et al. Thrombin activity and platelet microparticle formation are increased in type 2 diabetic platelets: a potential correlation with caspase activation. Thromb Res 2002; 107: 217-221. 
78. Trzepizur W, et al. Microparticles and vascular dysfunction in obstructive sleep apnoea. Eur Respir J 2014; 44: 207-216.

79. Sabatier F, et al. Type 1 and type 2 diabetic patients display different patterns of cellular microparticles. Diabetes 2002; 51: 2840-2845.

80. Preston RA, et al. Effects of severe hypertension on endothelial and platelet microparticles. Hypertension 2003; 41: 211-217.

81. Murakami T, et al. Impact of weight reduction on production of platelet-derived microparticles and fibrinolytic parameters in obesity. Thromb Res 2007; 119: 45-53.

82. Helal O, et al. Increased levels of microparticles originating from endothelial cells, platelets and erythrocytes in subjects with metabolic syndrome: relationship with oxidative stress. Nutr Metab Cardiovasc Dis 2011; 21: 665-671.

83. Suades R, et al. Lipid-lowering therapy with statins reduces microparticle shedding from endothelium, platelets and inflammatory cells. Thromb Haemost 2013; 110: 366-377.

84. Suades R, et al. Circulating CD45+/CD3+ lymphocyte-derived microparticles map lipid-rich atherosclerotic plaques in familial hypercholesterolaemia patients. Thromb Haemost 2014; 111: 111-121.

85. Mobarrez F, et al. Atorvastatin reduces thrombin generation and expression of tissue factor, P-selectin and GPIIIa on platelet-derived microparticles in patients with peripheral arterial occlusive disease. Thromb Haemost 2011; 106: 344-352.

86. Jayachandran $\mathrm{M}$, et al. Characterisation of blood borne microparticles as markers of premature coronary calcification in newly menopausal women. Am J Physiol Heart Circ Physiol 2008; 295: H931-H938.

87. Mause SF. Platelet microparticles: reinforcing the hegemony of platelets in atherothrombosis. Thromb Haemost 2013; 109: 5-6.

88. Merten M, et al. Platelet microparticles promote platelet interaction with subendothelial matrix in a glycoprotein IIb/IIIa-dependent mechanism. Circulation 1999; 99: 2577-2582.

89. Suades R, et al. Circulating and platelet-derived microparticles in human blood enhance thrombosis on atherosclerotic plaques. Thromb Haemost 2012; 108: 1208-1219.

90. Agouni A, et al. Endothelial dysfunction caused by circulating microparticles from patients with metabolic syndrome. Am J Pathol 2008; 173: 1210-1219.

91. Agouni A, et al. Microparticles from patients with metabolic syndrome induce vascular hypo-reactivity via Fas/Fas-ligand pathway in mice. PLoS one 2011; 6: e27809.

92. Hansson GK, et al. Detection of activated T lymphocytes in the human atherosclerotic plaque. Am J Pathol 1989; 135: 169-175.

93. Sadallah S, et al. Ectosomes released by platelets induce differentiation of CD4+T cells into T regulatory cells. Thromb Haemost 2014; 112: 1219-1229.

94. Weber A, et al. Platelet-derived microparticles stimulate coronary artery smooth muscle cell mitogenesis by a PDGF-independent mechanism. Thromb Res 2000; 98: 461-466.

95. Butoi ED, et al. Cross talk between smooth muscle cells and monocytes/activated monocytes via CX3CL1/CX3CR1 axis augments expression of pro-atherogenic molecules. Biochim Biophys Acta 2011; 1813: 2026-2035.

96. Boilard E, et al. Platelets amplify inflammation in arthritis via collagen-dependent microparticle production. Science 2010; 327: 580-583.

97. Jancar S, Sanchez Crespo M. Immune complex-mediated tissue injury: a multistep paradigm. Trends Immunol 2005; 26: 48-55.

98. Mayadas TN, et al. Mechanisms of immune complex-mediated neutrophil recruitment and tissue injury. Circulation 2009; 120: 2012-2024.

99. Nielsen CT, et al. Increased IgG on cell-derived plasma microparticles in systemic lupus erythematosus is associated with autoantibodies and complement activation. Arthritis Rheum 2012; 64: 1227-1236.

100. van Eijk IC, et al. Circulating microparticles remain associated with complement activation despite intensive anti-inflammatory therapy in early rheumatoid arthritis. Ann Rheum Dis 2010; 69: 1378-1382.
101. Cloutier N, et al. The exposure of autoantigens by microparticles underlies the formation of potent inflammatory components: the microparticle-associated immune complexes. EMBO Mol Med 2013; 5: 235-249.

102. Rozmyslowicz T, et al. Platelet- and megakaryocyte-derived microparticles transfer CXCR4 receptor to CXCR4-null cells and make them susceptible to infection by X4-HIV. AIDS 2003; 17: 33-42.

103. Mause SF, et al. Platelet microparticles enhance the vasoregenerative potential of angiogenic early outgrowth cells after vascular injury. Circulation 2010; 122: 495-506.

104. Risitano A, et al. Platelets and platelet-like particles mediate intercellular RNA transfer. Blood 2012; 119: 6288-6295.

105. Diehl P, et al. Microparticles: major transport vehicles for distinct microRNAs in circulation. Cardiovasc Res 2012; 93: 633-644.

106. Laffont B, et al. Activated platelets can deliver mRNA regulatory Ago2*microRNA complexes to endothelial cells via microparticles. Blood 2013; 122: 253-261.

107. Barry OP, et al. Modulation of monocyte-endothelial cell interactions by platelet microparticles. J Clin Invest 1998; 102: 136-144.

108. Vasina EM, et al. Microparticles from apoptotic platelets promote resident macrophage differentiation. Cell Death Dis 2011; 2: e211.

109. Rautou PE, et al. Microparticles from human atherosclerotic plaques promote endothelial ICAM-1-dependent monocyte adhesion and transendothelial migration. Circ Res 2011; 108: 335-343.

110. Sadallah S, et al. Microparticles (ectosomes) shed by stored human platelets downregulate macrophages and modify the development of dendritic cells. J Immunol 2011; 186: 6543-6552.

111. Vasina EM, et al. Aging- and activation-induced platelet microparticles suppress apoptosis in monocytic cells and differentially signal to proinflammatory mediator release. Am J Blood Res 2013; 3: 107-123.

112. Faure V, et al. Elevation of circulating endothelial microparticles in patients with chronic renal failure. J Thromb Haemost 2006; 4: 566-573.

113. Vion AC, et al. Shear stress regulates endothelial microparticle release. Circ Res 2013; 112: 1323-1333.

114. Zernecke A, et al. Delivery of microRNA-126 by apoptotic bodies induces CXCL12-dependent vascular protection. Sci Signal 2009; 2: ra81.

115. Abid Hussein MN, et al. Phospholipid composition of in vitro endothelial microparticles and their in vivo thrombogenic properties. Thromb Res 2008; 121: 865-871.

116. Dignat-George F, Boulanger CM. The many faces of endothelial microparticles. Arterioscler Thromb Vasc Biol 2011; 31: 27-33.

117. Abid Hussein MN, et al. Inhibition of microparticle release triggers endothelial cell apoptosis and detachment. Thromb Haemost 2007; 98: 1096-1107.

118. Mezentsev A, et al. Endothelial microparticles affect angiogenesis in vitro: role of oxidative stress. Am J Physiol Heart Circ Physiol 2005; 289: H1106-1114.

119. Leroyer AS, et al. Microparticles from ischemic muscle promotes postnatal vasculogenesis. Circulation 2009; 119: 2808-2817.

120. Habersberger J, et al. Circulating microparticles generate and transport monomeric C-reactive protein in patients with myocardial infarction. Cardiovasc Res 2012; 96: 64-72.

121. Wang JG, et al. Monocytic microparticles activate endothelial cells in an IL1beta-dependent manner. Blood 2011; 118: 2366-2374.

122. Mallat Z, et al. Shed membrane microparticles with procoagulant potential in human atherosclerotic plaques: a role for apoptosis in plaque thrombogenicity. Circulation 1999; 99: 348-353.

123. Leroyer AS, et al. Cellular origins and thrombogenic activity of microparticles isolated from human atherosclerotic plaques. J Am Coll Cardiol 2007; 49: 772-777.

124. Kapustin AN, et al. Vascular Smooth Muscle Cell Calcification is Mediated by Regulated Exosome Secretion. Circ Res 2015; 116: 1312-1323. 